BIOMEDICA

Vol. 8 , Nos. 1 y $2-1988$

\title{
COMPORTAMIENTO DE FLAGELADOS DE LA FAMILIA TRYPANOSOMATIDAE EN DOS MEDIOS DE CULTIVO MODIFICADOS
}

\author{
SOFIA DUQUE BELTRAN*, ELVIA CACERES VEGA**, AUGUSTO CORREDOR ARJONA***
}

Se reporta un medio de cultivo monofásico sólido (NNN modificado) y un medio monofásico líquido (RE I modificado) de gran utilidad para cultivos in vitro de flagelados de la Familia Trypanosomatidae. El medio NNN modificado mostró sostener la población de flagelados por un tiempo más prolongado y en mayor número que el medio clásico. Las cepas de Leishmania braziliensis panamensis y Leishmania mexicana amazonensis se adaptaron mejor en el medio modificado. En el medio RE I modificado la especie Trypanosoma rangeli se adaptó mejor que las cepas Trypanosoma cruzi y Crithidia luciliae.

\section{INTRODUCCION}

Los medios de cultivo usados hasta hoy para flagelados son modificaciones de medios difásicos de agar sangre o medios líquidos de diferentes composiciones $(1,2,3,4,5,6)$.

Uno de los primeros medios desarrollados y de amplio uso en el aislamiento y mantenimiento de flagelados del género Leishmania es el NNN que fundamentalmente es un agar sangre de composición simple (5, 6). Al medio NNN se le han hecho desde su divulgación muchas modificaciones tanto en la fase sólida como en la líquida $(7,8,9,10,11)$. Varios medios de cultivo han sido introducidos para el cultivo de flagelados de la Familia Trypanosomatidae con variadas composiciones $(1,2,12,13,14,15,16,17,18$, 19 y 20$)$.

El presente trabajo presenta la modificación del medio NNN $(5,6)$ y del RE I (3).

\section{MATERIALES Y METODOS}

1. Parásitos y medios de cultivo

Los flagelados utilizados son leishmanias de las subespecies braziliensis panamensis y mexicana ama- zonensis; tripanosomas cruzi y rangeli y Crithidia luciliae. Las leishmanias provinieron de aislamientos de pacientes. Los tripanosomas de Rhodnius prolixus y Aotus sp. respectivamente y la cepa Crithidia luciliae fue suministrada por el laboratorio de Parasitología de la Universidad Industrial de Santander.

Composición de los medios NNN modificado

Elementos: gramos $/ 100 \mathrm{ml}$

Agar nutritivo

1.8

$\mathrm{NaCl}$

0.6

Glucosa

0.6

$\mathrm{Na}_{2} \mathrm{HPO}_{4} \cdot 12 \mathrm{H}_{2} \mathrm{O}$

0.006

$\mathrm{KH}_{2} \mathrm{PO}^{4}$

0.006

Infusión cerebro corazón

2.12

Agua destilada estéril

$100.00 \mathrm{ml}$

Mezclar bien y ajustar pH a 7.2 con $\mathrm{NaOH} 1 \mathrm{~N}$ o $\mathrm{HCl} 1 \mathrm{~N}$. Esterilizar el medio a $121^{\circ} \mathrm{C}$ durante $15 \mathrm{mi}-$ nutos. Dejar enfriar hasta $45^{\circ} \mathrm{C}$ y en condiciones asépticas agregar sangre desfibrinada de conejo o en su defecto sangre humana preferiblemente grupo $\mathrm{O} R \mathrm{Rh}$

\footnotetext{
* Bióloga, Grupo de Parasitología, Instituto Nacional de Salud.

** Bacterióloga, Grupo de Parasitología, Instituto Nacional de Salud.

* * *Médico, Jefe Grupo de Parasitología, Instituto Nacional de Salud; Profesor, Facultad de Medicina, Universidad Nacional de Colombia.
} 
positivo hasta una concentración final del 10\% (v/v), libre de cualquier tipo de contaminación. Distribuir volúmenes de $3 \mathrm{ml}$ en tubos tapa rosca de $(13 \times 100$ $\mathrm{mm}$ ) estériles e inclinarlos de tal manera que el medio no llegue a más de la mitad del tubo dejando $1 \mathrm{ml}$ de base. Una vez solidificado el medio, colocarlo a $4^{\circ} \mathrm{C}$ para la obtención de agua de condensación. Realizar pruebas de esterilidad.

\section{RE I modificado}

Elementos:

gramos/1000 ml

$\mathrm{NaCl}$

8.0

$\mathrm{KCl}$

0.4

$\mathrm{MgSO}_{4} \cdot 7 \mathrm{H}_{2} \mathrm{O}$

0.2

$\mathrm{Na}_{2} \mathrm{HPO}_{4} 12 \mathrm{H}_{2} \mathrm{O}$

0.06

$\mathrm{KH}_{2} \mathrm{PO}_{4}$

0.06

$\mathrm{CaCl}_{2}$

Glucosa

$\mathrm{NaHCO}_{3}$

Infusión cerebro corazón

Agua destilada estéril

$1000.00 \mathrm{ml}$

Mezclar bien y ajustar $\mathrm{pH}$ a 7.2 con $\mathrm{NaOH} 1 \mathrm{~N}$ o $\mathrm{HCl} 1 \mathrm{~N}$. Esterilizar el medio a $121^{\circ} \mathrm{C}$ durante $15 \mathrm{mi}-$ nutos. Agregar hasta una concentración final del $2 \%$ (v/v) suero bovino estéril inactivado a $56^{\circ} \mathrm{C}$ durante 30 minutos. Realizar pruebas de esterilidad.

Se usaron los medios NNN clásico $(5,6)$ y NNN modificado adicionados con solución salina fisiológica para el cultivo de flagelados del género Leishmania, Trypanosoma cruzi y Crithidia luciliae fueron cultivados en Maekelt (21) y el Trypanosoma rangeli en Tobie modificado (22) adicionados con RE I modificado y fueron empleadas como control las fases sólidas anteriormente mencionadas, adicionadas con solución salina fisiológica.

\section{Determinación del crecimiento poblacional}

El comportamiento de los flagelados en los medios de cultivo anteriormente mencionados fue llevado a cabo mediante cuantificaciones periódicas de las po- blaciones por $\mathrm{ml}$, partiendo para cada cépa de inóculos de poblaciones por $\mathrm{ml}$ conocidos.

\section{Análisis estadístico}

Los resultados fueron analizados mediante Test " $\mathrm{t}$ " de Student a un nivel de significancia del 5\%.

\section{RESULTADOS}

\section{Leishmania braziliensis panamensis}

Su crecimiento poblacional en el medio de cultivo NNN modificado es mejor al obtenido en NNN clásico (Fig. 1), observándose después del segundo día en el medio NNN clásico un descenso en la población con una recuperación al cuarto día, para luego presentarlo nuevamente, muy marcado hacia el octavo y décimo día. A diferencia, el comportamiento en el medio de cultivo NNN modificado muestra al décimo día una población superior a la obtenida en NNN clásico. La Leishmania braziliensis panamensis comienza con un ascenso de población desde el segundo día continuándolo a través de su cuarto y octavo día para luego permanecer estable al décimo día donde presenta un buen crecimiento de población con respecto al obtenido en NNN clásico. Esta observación nos permite sugerir que los repiques, si se continúa empleando NNN clásico, se tendrían que llevar a cabo cada cuatro días, mientras que en NNN modificado se efectuaría cada ocho días con la obtención de una población mayor y más estable de parásitos. Para los recuentos llevacios a cabo en el segundo, octavo y décimo día de crecimiento, las diferencias fueron estadísticamente significativas (Tabla 1).

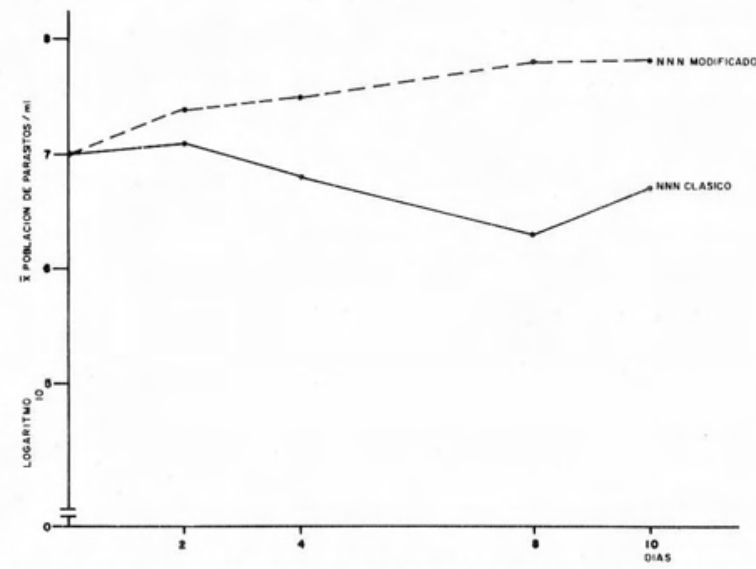

Fig. 1. Leishmania braziliensis panamensis NNN clásico Vs. NNN modificado. 
COMPORTAMIENTO DE FLAGELADOS DE LA FAMILIA TRYPANOSOMATIDAE EN DOS MEDIOS DE CULTIVO MODIFICADOS

TABLA 1. TEST DE STUDENT

\begin{tabular}{|c|c|c|c|c|c|c|c|c|}
\hline \multirow{3}{*}{$\begin{array}{l}\text { FLAGELADOS } \\
\text { FA MILIA } \\
\text { TRYPANOSOMA - } \\
\text { TIDAE }\end{array}$} & \multirow{2}{*}{\multicolumn{2}{|c|}{$\begin{aligned} \text { NNN CLASICO+ } & \text { Solución } \\
& \text { Salina } \\
& \text { Fisiológica }\end{aligned}$}} & \multirow{2}{*}{\multicolumn{2}{|c|}{$\begin{aligned} \text { NNN MODIFICADO+ } & \text { Solución } \\
& \text { Salina } \\
& \text { Fisiológico }\end{aligned}$}} & \multirow{3}{*}{$\begin{array}{l}\text { Tiempo } \\
\text { dios }\end{array}$} & \multirow{3}{*}{$\begin{array}{c}\text { Número } \\
\text { de } \\
\text { muestras } \\
\text { cado } \\
\text { medio }\end{array}$} & \multicolumn{2}{|c|}{\begin{tabular}{|l} 
TEST DE \\
STUDENT \\
\end{tabular}} \\
\hline & & & & & & & & Significancid \\
\hline & Promedio $\frac{\log .10}{x}$ & $\begin{array}{l}\text { Desviación } \\
\text { standard } \\
\text { log. } 10\end{array}$ & $\underset{\bar{x}}{\text { Promedio }} \log .10$ & $\begin{array}{l}\text { Desviación } \\
\text { standard } \\
\text { log. } 10\end{array}$ & & & dos. & $(p<0.05)$ \\
\hline \multirow{4}{*}{$\begin{array}{l}\text { Leishmania } \\
\text { braziliensis } \\
\text { ponamensis }\end{array}$} & 6.34 & 0.14 & 6.72 & 0.18 & 2 & 4 & 2.87 & si \\
\hline & 6.73 & 0.20 & 6.90 & 0.11 & 4 & 4 & 1.25 & No \\
\hline & 6. 18 & 0.21 & 6.99 & 0.09 & 8 & 4 & 6.20 & sí \\
\hline & 6. 19 & 0.32 & 7.05 & 0.09 & 10 & 4 & 4.49 & sí \\
\hline \multirow{5}{*}{$\begin{array}{l}\text { Leishmania } \\
\text { mexicana } \\
\text { amazonensis }\end{array}$} & \multicolumn{2}{|c|}{$\begin{aligned} \text { NNN CLASICO }+ & \text { Solina } \\
& \text { Fisiológica }\end{aligned}$} & \multicolumn{2}{|c|}{$\begin{aligned} \text { Solución } \\
\text { NNN MODIFICADO + }\end{aligned}$} & & & & \\
\hline & 7. 14 & 0.11 & 7.45 & 0.01 & 2 & 4 & 4.88 & sí \\
\hline & 6.72 & 0.18 & 7.51 & 0.02 & 4 & 4 & 7.33 & sí \\
\hline & 6.37 & 0.18 & 7.73 & 0.04 & 8 & 4 & 13.11 & si \\
\hline & 6.69 & 0.12 & 7.73 & 0.07 & 10 & 4 & 12 & si \\
\hline \multirow{6}{*}{ Trypanosoma } & MAEKELT + & $\begin{array}{l}\text { Solución } \\
\text { Salina } \\
\text { Fisiológica }\end{array}$ & \multicolumn{2}{|l|}{ MAEKELT + } & & & \\
\hline & 6.54 & 5.73 & 6.62 & 5.57 & 1 & 4 & 1.82 & No \\
\hline & 6.75 & 6.03 & 6.87 & 6.09 & 4 & 4 & 1.83 & No \\
\hline & 7.41 & 6.75 & 7.53 & 6.73 & 6 & 4 & 1.73 & No \\
\hline & 7.88 & 7.43 & 8.01 & 7.27 & 8 & 4 & 1.36 & No \\
\hline & 8.41 & 7.47 & 8.51 & 7.71 & 12 & 4 & 1.96 & No \\
\hline & TOBIE & $\begin{array}{l}\text { Solución } \\
\text { Salina } \\
\text { Fisiológica }\end{array}$ & TOBIE & $\begin{array}{c}\mathrm{REI} \\
\text { modificado }\end{array}$ & & & & \\
\hline Trypanosoma & 6.98 & 6.45 & 7.27 & 5.93 & 1 & 4 & 5.54 & Si \\
\hline rangell & 7.26 & 6. 39 & 7.59 & 6.79 & 4 & 4 & 5.46 & sí \\
\hline & 7.53 & 6.85 & 7.87 & 7.29 & 6 & 4 & 4.03 & Sí \\
\hline & 6.70 & 6.33 & 6.97 & 6.67 & 8 & 4 & 1.43 & No \\
\hline Crithidia & MAEKELT + & $\begin{array}{l}\text { Solución } \\
\text { Salina } \\
\text { Fisiológica }\end{array}$ & MAEKELT + & $\begin{array}{c}\mathrm{REI} \\
\text { modificado }\end{array}$ & & & & \\
\hline & 8.00 & 7.00 & 8.26 & 7.80 & 1 & 4 & 2.14 & No \\
\hline Luciliae & 8.32 & 7.46 & 8.41 & 7.84 & 3 & 4 & 1.02 & No \\
\hline & 8.73 & 8. 22 & 8.86 & 7.73 & 5 & 4 & 1.83 & No \\
\hline
\end{tabular}




\section{Leishmania mexicana amazonensis}

La tasa de crecimiento es mayor en el medio de cultivo NNN modificado que en el medio de cultivo NNN clásico. En este último los parásitos alcanzan un leve incremento de población al segundo día para luego disminuir drásticamente, alcanzando otro pequeño incremento de población al décimo día, siendo la población total final muy inferior a la población inicial de parásitos. El complejo Leishmania mexicana siempre presenta incrementos poblacionales en NNN modificado a través del tiempo, tratando de estabilizarse al octavo día; por lo tanto, podríamos decir que un repique puede ser llevado a cabo al octavo día con buenos resultados (Fig. 2).

Para los recuentos llevados a cabo en el segundo, cuarto, octavo y décimo día de crecimiento las diferencias fueron estadísticamente significativas (Tabla 1).

\section{Trypanosoma cruzi}

Los flagelados tanto en solución salina fisiológica como en RE I modificado tuvieron una población similar en los días uno, cuatro, seis, ocho y doce (Fig. 3). No hubo diferencias estadísticamente significativas en el crecimiento entre estos medios con esta especie (Tabla 1).

\section{Trypanosoma rangeli}

Los tripanosomas crecieron progresivamente en los días uno, cuatro y seis en ambas fases líquidas pero con una mayor población en RE I modificado; pero, al octavo día, las poblaciones decrecieron en ambos medios. Se sugiere llevar a cabo pases para esta especie cada seis días en RE I modificado (Fig. 4).

Para los recuentos llevados a cabo en el primer, cuarto y sexto día de crecimiento, las diferencias fueron estadísticamente significativas (Tabla 1).

\section{Crithidia luciliae}

El incremento de población en el día primero con respecto al inóculo es muy grande, habiendo diferencia en el número de parásitos entre las fases líquidas (Fig. 5). Sin embargo, las diferencias en crecimiento no fueron estadísticamente significativas (Tabla 1).

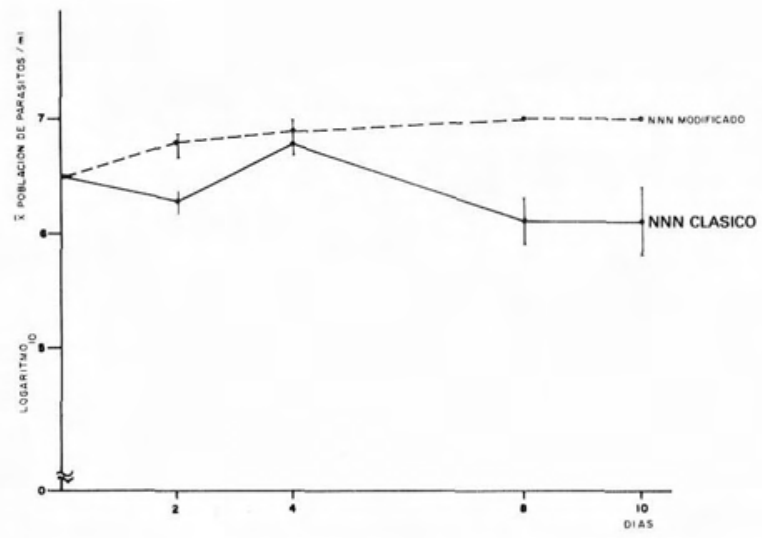

Fig. 2. Leishmania mexicana amazonensis NNN clásico Vs. NNN modificado.

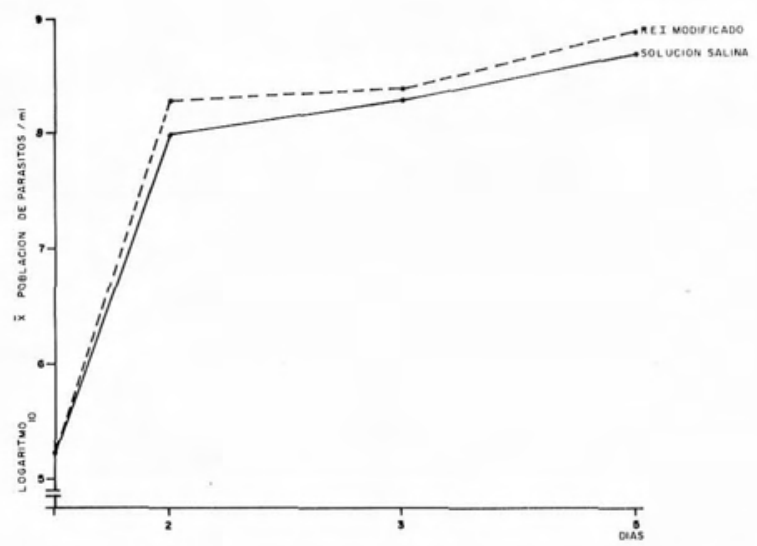

Fig. 3. Trypanosoma cruzi Maekelt + Solución Salina Vs. Maekelt + RE I modificado.

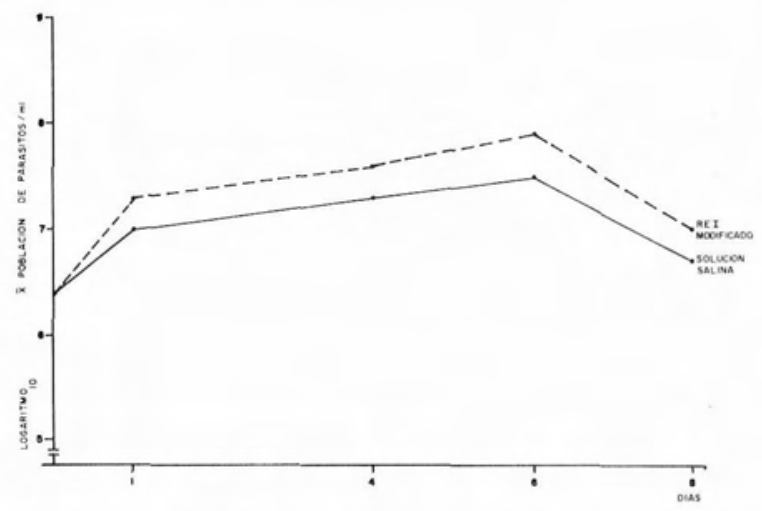

Fig. 4. Trypanosoma rangeli. Tobie + Solución Salina Vs. Tobie + RE I modificado. 


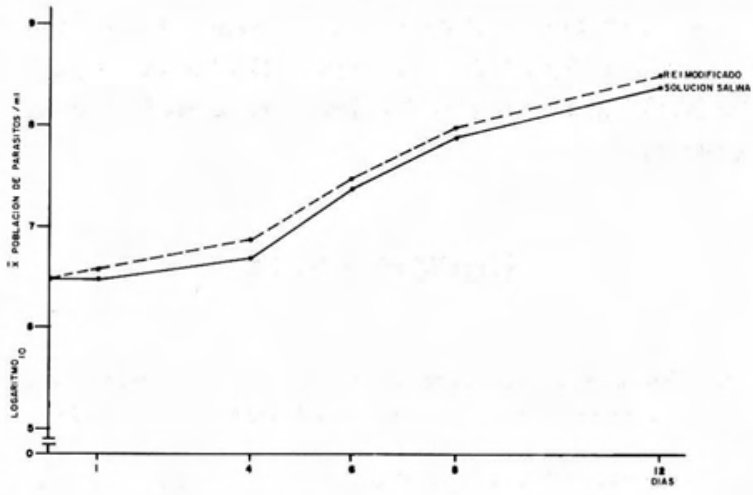

Fig. 5. Crithidia luciliae. Maekel + Solución Salina Vs. Maekel + RE I modificado.

\section{DISCUSION}

La mezcla de aminoácidos y vitaminas usada en el medio RE I descrito originalmente por Steiger y Steiger (3) fue sustituida por infusión cerebro corazón y $2 \%$ de suero bovino, el cual se agrega al medio, con anterioridad a la siembra. Las vitaminas no se adicionaron por haber mostrado en ensayos preliminares efectos adversos.

Con respecto al medio de NNN originalmente descrito por Novy y McNeal (5) y Nicolle (6) nuestra modificación consistió en agregar glucosa, fosfatos y enriquecer el medio con infusión cerebro corazón y agar nutritivo. Es indispensable la obtención de agua de condensación en este medio pues ella permite un mayor desarrollo de parásitos. Shaw y Lainson (23) observaron que el agua de condensación para la obtención de promastigotes es más efectiva que el medio de cultivo Schneider y aún mejor que el NNN clásico con solución salina o solución de Locke.

Muchos de los medios ampliamente utilizados para el cultivo de parásitos requieren de sangre total o de sólo glóbulos rojos $(1,2)$. El volumen de sangre empleada en la elaboración del medio NNN modificado variará según su contenido de hemoglobina. El contenido de hemoglobina que generalmente debe poseer el medio es de 15 gramos $/ 100 \mathrm{ml}$.

El suero fetal bovino provee los factores necesarios para el crecimiento y multiplicación de los parásitos, ya que posee proteínas mitogénicas que actúan sobre la membrana celular (24) y poblaciones mayores de parásitos pueden ser obtenidas (25). El suero bovino aunque no posee los mismos nutrientes que el suero bovino fetal, en el RE I modificado al $2 \%$ produce buena población de flagelados al sexto día para Trypanosoma rangeli y octavo día para Leishmania braziliensis panamensis y Leishmania mexicana amazonensis. Esto favorece los costos en la producción puesto que el suero bovino tiene un costo mucho menor que el suero bovino fetal.

Tanto para las sangres como para los sueros empleados en la elaboración de los medios se debe llevar a cabo el mismo control para contaminantes que el efectuado para los medios descritos anteriormente, ocho días antes de su preparación.

Los medios una vez se sirven en los tubos tapa rosca estériles o en frascos volumétricos de $100 \mathrm{ml}$ estériles, deben tener un control de microorganismos contaminantes muy estricto para asegurar una efectiva esterilidad. Los cultivos axénicos de parásitos son una herramienta muy valiosa para análisis in vitro e in vivo de propiedades tales como: infectividad, requerimientos metabólicos y variación antigénica (26).

Los medios ideados y elaborados por el Grupo de Parasitología del Instituto Nacional de Salud son fáciles de preparar y esterilizar, de costo relativamente bajo, en ellos se obtienen mayores poblaciones de flagelados que en otros ya experimentados como: $\mathrm{NNN}, 4 \mathrm{~N}$, etc. y facilitan el buen crecimiento de más de una especie de flagelados.

La diferencia entre las poblaciones de parásitos de Trypanosoma cruzi y Trypanosoma rangeli puede deberse no sólo a la presencia de fases sólidas diferente (Maekelt para Trypanosoma cruzi, Tobie modificado para Trypanosoma rangeli) sino también a la naturaleza y metabolismo diferente de cada una de las especies.

En el caso de Trypanosoma cruzi y de Crithidia luciliae se puede emplear como fase líquida para el mantenimiento de estos flagelados solución salina fisiológica ya que por ser un medio líquido tan pobre en nutrientes ofrece menos riesgos de contaminación y las poblaciones obtenidas son similares a las obtenidas con RE I modificado. 
Se recomienda elaborar los medios de cultivo NNN modificado y RE I modificado lo más exactamente posible, de acuerdo con la fórmula, ya que una mínima alteración en la concentración de los elementos altera el crecimiento en forma negativa (27). Además, la presencia de una fase líquida enriquecida con nutrientes propicios (RE I modificado) es fundamental para el buen desarrollo, mantenimiento y obtención de poblaciones numerosas.

Varias especies de Leishmania cutánea y mococutánea al igual que varias especies de Trypanosoma y Crithidia cultivadas en estos medios, han mantenido su viabilidad después de preservadas en nitrógeno líquido y su infectividad para animales de laboratorio.

Es preferible emplear sangre desfibrinada de conejos en buenas condiciones de salud y peso, con la concentración de hemoglobina óptima, que sangre humana.

\section{SUMMARY}

We report the results obtained with a monophasic solid medium (NNN modified) and a liquid monophasic medium (RE I modified) for in vitro culture of flagellates of the Family Trypanosomatidae. The modified NNN medium supported growth for a longer period of time and at higher population concentrations than did the clasic NNN medium. The strains of Leishmania brazilienzis panamensis and Leishmania mexicana amazonensis grew better in modified NNN medium. The strain of Trypanosoma rangeli grew better in modified RE I that the strains of Trypanosoma cruzi and Crithidia luciliae.

\section{AGRADECIMIENTOS}

A las siguientes personas del Instituto Nacional de Salud: doctor Luis Carlos Orozco V., Jefe del Grupo de Micobacterias, por sus sugerencias en los aspectos bacteriológicos y estadísticos, doctor Ruben Santiago Nicholls O., Médico del Grupo de Parasitología, por su ayuda en los análisis estadísticos y comentarios. Al Grupo de Control de Calidad especialmente a Martha Rodríguez por los análisis hematológicos. A Dioselina Peláez C., Bacterióloga, por la preparación de los medios de cultivo.
Este trabajo se realizó con los auspicios del Instituto Nacional de Salud de Colombia y del Grant AI-2018 del National Institute of Health de Estados Unidos de América.

\section{BIBLIOGRAFIA}

1. Dwyer DM. A monophasic medium for cultivating Leishmania donovani in large numbers. J Parasitol 1972; 58: 847.

2. Mansour, Hady J, McConnell E. A modified liquid medium for Leishmania. J Parasitol 1973; 59: 1088.

3. Steiger RF, Steiger E. A defined medium for cultivating Leishmania donovani and L. braziliensis. J Parasitol 1976; 62: 1010 .

4. Marin F, García de Lomas J, Peñarrubia MPG, Pañalver J. Cultivation of Leishmania: comparison of different media for promastigote cultivation. Ann Trop Med Parasitol 1982; 76: 607.

5. Novy FG, MacNeal WJ. On the cultivation of Trypanosoma brucei. J Infect Dis 1904; 1: 1.

6. Nicolle Ch. Culture du parasite du Bouton D'Orient. Comp Rend Heb Sea Acad Sci Paris 1904; 146: 842.

7. Mathis C. Sur une modification au milieu de Novy MacNeal pour la culture des trypanosomes. Comp Rend Sea Soc Biol 1906; 61: 550.

8. Weinman D. Cultivation of the African sleeping sickness trypanosomes from the blood and cerebro-spinal fluid of patients and suspects. Trans R Soc Trop Med Hyg 1960; 54: 180.

9. Tobie EJ. The cultivation of Trypanosoma congolense in vitro. J Parasitol 1958; 44: 241.

10. Jadin J, Wery M. La culture des Trypanosomidae. Ann Soc Belge Med Trop 1963; 43: 831.

11. Benex J, Lamy L. Isolement, characterisation et fractionement d'un antigéne Leishmanien pour réaction de fixation du complément et test de précipitation. Ann Inst Pasteur 1967; 91.

12. Jadin J, Le Ray D. Acquisitions dans les techniques de culture des trypanosomes africains. Ann Soc Belge Med Trop 1969; 49: 331 .

13. Le Ray D, Afchain D, Jadin J, Capron A, Yasarol S, Lanotte G, Fameree L. Diagnostic inmunoelectroforetique de la Leishmaniose viscerale á l'aide d'un estrait antigenique hydrosoluble de Leishmania donovani. Results préliminares. Ann Soc Belge Med Trop 1973; 53: 31. 
14. Feinberg JG, Whittington MJ. A culture medium for Trichomonas vaginalis Donné and species of Candida. J Clin Path 1957; 10: 327.

15. Schnur LF, Zuckerman A, Greenblatt ChL. Leishmanial serotypes as distinguished by the gel diffusion of factors excreted in vitro and in vivo. Israel J Med Sci 1972; 8: 932.

16. Rezai HR, Behforouz N, Amirhakimi GH, Konanteb J. Immunofluorescence and counter immunoelectrophoresis in the diagnosis of Kala-azar. Trans R Soc Trop Med Hyg 1977; 71: 149.

17. Frah FS, Samra SA, Nuwayri- salti N. The role of the macrophage in cutaneous leishmanisis. Immunology. 1975; 29: 755.

18. Chance ML, Peters W, Shchory L. Biochemical taxonomy of Leishmania: I. Observations on DNA. Ann Trop Med Parasitol 1974; 68: 307.

19. Berens RL, Brun R, Krassner SM. A simple monophasic medium for axenic culture of hemoflagellates. J Parasitol 1976; $62: 360$.

20. Hendricks LD, Wood DE, Hajduc ME. Haemoflagellates: commercially available liquid media for rapid cultivation. Parasitology 1978; 76: 309.
21. Maekelt GA. Die komplementbindugsreaktion der Chagas Krankheit. Z Tropenmede Parasitol 1960; 11: 152.

22. Sánchez ME, Ramírez RS. La posible diferenciación de las infecciones causadas por los Trypanosomas cruzi y rangeli por la técnica inmunoenzimática ELISA. Tesis de grado. Universidad Nacional de Colombia. Facultad de Ciencias, Departamento de Química.

23. Shaw JJ, Lainson R. The in vitro cultivation of members of the Leishmania braziliensis complex. Trans R Soc Trop Med Hyg 1981; 75: 127.

24. O'Daly JA. Serum proteins promoting $\left({ }^{3} \mathrm{H}\right)$ thymidine uptake by Trypanosoma (Schizotrypanum) cruzi (Chagas) in vitro. J Protozool 1975; 22: 550.

25. Hart DT, Vickerman K, Coombs GH. Transformation in vitro of Leishmania mexicana amastigotes to promastigotes: nutritional requirements and the effect of drugs. Parasitology 1981; 83: 529.

26. Taylor AER, Baker JR. Cultivation of parasites in vitro. Blackwell Scientific Publications. Oxford. 1968.

27. Walton BC, Shaw JJ, Lainson R. Observations on the in vitro cultivation of Leishmania braziliensis. J Parasitol 1977; 63: 1118 . 